\title{
APRENDIZAJE LÉXICO DE UNA LENGUA EXTRANJERA MEDIANTE UNA METODOLOGÍA BASADA EN LA ADQUISICIÓN DE CONTENIDOS ${ }^{1}$
}

\author{
Luisa $\mathrm{M}^{\mathrm{a}}$ González Rodríguez y María Jesús Sánchez Manzano²
}

\begin{abstract}
Resumen: Este trabajo se propone reflexionar sobre una serie de aspectos relacionados con las últimas investigaciones centradas en el aprendizaje léxico en una lengua extranjera y analizar cómo todos estos aspectos pueden ser integrados mediante la aplicación de la metodología basada en contenidos con la finalidad de incrementar el repertorio léxico de los alumnos de forma natural, significativa y duradera.
\end{abstract}

Palabras clave: Aprendizaje, léxico, lengua extranjera, instrucción, desarrollo léxico, enseñanza basada en contenidos.

\begin{abstract}
The aim of this paper is to refocus attention on some aspects connected to the latest research on lexical learning in a foreign language and to analyze how all those basic and fundamental aspects can be integrated by using Content-based Instruction in order to improve our students' lexicon in a natural, meaningful way.

Key words: Vocabulary, learning, foreign language, instruction, vocabulary development, content-based instruction.
\end{abstract}

\section{INTRODUCCIÓN}

El aprendizaje de una lengua basado en los contenidos es un enfoque integral que considera que la utilización de contenidos relevantes contribuye tanto a la mejora en la competencia lingüística como al desarrollo cognitivo, académico y social. Esta metodología se basa en la premisa de que la lengua es un medio para aprender contenidos y los contenidos son un recurso para aprender la lengua. Por este motivo, se utilizan temas de otras disciplinas con el propósito de aprender la lengua inglesa mientras se aprenden contenidos académicos. Se elimina así la separación artificial entre forma y contenido ya que los objetivos lingüísticos se consiguen mediante el aprendizaje de contenidos y viceversa.

Debido a que esta metodología pretende que los alumnos adquieran contenidos mediante el uso de una lengua extranjera, parece necesario conocer un elevado porcentaje de vocabulario que facilite la comprensión del discurso oral y escrito. Se parte de la base de que sin un adecuado repertorio léxico los alumnos serán incapaces de procesar el input a partir del cual adquieren los contenidos. De hecho, la mayoría de los lingüistas coinciden en la importancia del repertorio léxico para la comprensión y producción del discurso (Bar-

\footnotetext{
$1 \quad$ Fecha de recepción: febrero 2008.

Fecha de aceptación y versión final: junio 2008.

2 Luisa $\mathrm{M}^{\mathrm{a}}$ González Rodríguez es Profesora Colaboradora y María Jesús Sánchez Manzano es Profesora Titular de Universidad, Departamento de Filología Inglesa, Universidad de Salamanca; $₫$ mjs@usal.es.
} 
croft 2004; Nation y Newton 2000; Stoller y Grabe 1995). Existen, además, estudios que relacionan el nivel de comprensión con el porcentaje de léxico conocido y que consideran necesario conocer más del $95 \%$ del vocabulario para comprender bien un texto y aplicar las estrategias de lectura de la lengua materna (Bonk 2000; Hu y Nation 2000; Laufer 2000; Waring 2002). Con respecto al discurso oral, las conclusiones de investigadores como Adolphs y Schmitt (2003) parecen coincidir también en la relación entre el repertorio léxico y el nivel de comprensión oral.

Partiendo de todas estas consideraciones sobre la correlación entre el repertorio léxico y la comprensión y producción del discurso, se pretende analizar los beneficios de la utilización de una metodología basada en el aprendizaje de contenidos para el aprendizaje del vocabulario. Este método permite enfocar el aprendizaje léxico desde una perspectiva de procesamiento cognitivo siguiendo las nuevas teorías sobre la adquisición de una segunda lengua (Brown, Sagers y LaPorte 1999; Ellis y He 1999). Se considera, además, que es una metodología que proporciona un enfoque interactivo de la instrucción léxica, ya que permite a los alumnos descubrir el significado de los términos desconocidos y, además, favorece el uso inmediato del nuevo léxico y su posterior integración en el sistema cognitivo del alumno.

Existen algunas teorías que se ocupan de explorar la mejor forma de aprender el vocabulario de una segunda lengua. Entre estas teorías destacamos las siguientes: el aprendizaje en contexto, el aprendizaje incidental mediante la lectura y la escucha extensiva, la instrucción explícita, el desarrollo de estrategias de deducción y memorización, la interacción de los alumnos mediante el uso de actividades comunicativas y, por último, la organización cognitiva o teoría de los esquemas aplicada al aprendizaje léxico. Debido a que la mayoría de estos aspectos sobre el aprendizaje léxico se han trabajado siempre de forma aislada, se pretende en este trabajo resaltar la importancia de utilizar una metodología que integre todas estas variables con el fin de conseguir mayores beneficios respecto al aprendizaje y uso del vocabulario en otro idioma. Analizaremos a continuación cada uno de estos principios teóricos que contribuyen al aprendizaje léxico teniendo en cuenta su posible aplicación e integración dentro de la metodología basada en el aprendizaje de contenidos.

\section{EL APRENDIZAJE LÉXICO EN CONTEXTO}

La mejor forma de aprender vocabulario es que los alumnos reciban un input rico y adecuado en contextos comprensibles. La mayoría de los investigadores (Kruse 1979; Nation 1990; Webb 2007a) parecen estar de acuerdo en que se aprende mejor el vocabulario dentro de un contexto, ya que el aprendizaje de palabras aisladas no ayuda a comprender su significado en profundidad. El contexto proporciona las pistas suficientes para adivinar el significado de las palabras desconocidas, ayuda a recordar mejor el vocabulario aprendido y establece conexiones entre los conocimientos previos y el vocabulario nuevo. Huckin y Haynes (1995) afirman que el contexto ofrece a los alumnos una riqueza de matices sobre el uso apropiado de la palabra, sus diferentes significados, las palabras con las que suele asociarse, etc. que la instrucción explícita por sí sola no podría ofrecer.

Por otra parte, si bien es verdad que el contexto ofrece una perspectiva multidimensional del léxico, también es necesario recordar que encontrar una palabra en un solo contexto no da la suficiente información para conocer su significado en profundidad. Para acceder al 
significado completo es necesario encontrar la palabra repetidamente en diferentes contextos (Schmitt y Carter 2000; Waring 2002). Se podría decir que la competencia léxica mejora gracias al aprendizaje en contexto que favorece los encuentros significativos que conducen a la integración del vocabulario en el repertorio léxico del alumno. Por este motivo, Brown (1995) recomienda que se tengan repetidos contactos con el léxico nuevo en contextos significativos y que se recicle el vocabulario aprendido poco después de su aprendizaje.

La metodología basada en contenidos parte de la idea de que la lectura de textos diferentes sobre un mismo tema favorece la posibilidad de encontrar el mismo vocabulario con cierta frecuencia en contextos variados y proporciona un conocimiento más profundo de esos conceptos (Brinton, Snow y Wesche 2004). Para ello, Schmitt y Carter (2000) proponen realizar una lectura restringida, es decir leer durante un tiempo sobre el mismo tema, debido a que permite a los alumnos familiarizarse con el tema y, consiguientemente, contribuye a facilitar tanto la lectura como el aprendizaje del léxico. Estos mismos beneficios se obtienen con la metodología basada en el aprendizaje de contenidos ya que el input (tanto oral como escrito) gira en torno a un mismo tema durante un largo período de tiempo, permitiendo la retención del vocabulario gracias a los encuentros frecuentes del mismo.

La enseñanza de una segunda lengua basada en el aprendizaje de contenidos proporciona la posibilidad de encontrar las mismas palabras repetidamente para comprender en profundidad los significados y usos de esos términos. Al contextualizar el aprendizaje del vocabulario, esta metodología es bastante eficaz desde un punto de vista pedagógico ya que el aprendizaje léxico es paralelo al proceso de lectura y la variedad de contextos proporciona una aproximación más profunda a los usos y significados del vocabulario. Se favorece, por tanto, un aprendizaje multidimensional del vocabulario ya que no sólo se aprende el significado referencial, sino también las relaciones paradigmáticas y sintagmáticas. Los repetidos encuentros permiten, además, tener en cuenta experiencias anteriores y construir el significado de forma más integrada y duradera. Es decir, el aprendizaje léxico se convierte en una experiencia de "internalización" que supone un conocimiento del mismo que va más allá de la aceptación pasiva del significado o de la mera memorización.

\section{APRENDIZAJE INCIDENTAL}

Algunas investigaciones reconocen que durante la lectura suele aprenderse gran cantidad de vocabulario de manera incidental (Coady 2000; Elley y Mangubhai 1983) y, por ello, muchos investigadores recomiendan abiertamente la lectura extensiva para desarrollar la competencia léxica (Krashen 1989; Grabe y Stoller 2000). Gass (1999) admite que la adquisición incidental, como consecuencia de otros procesos cognitivos relacionados con la comprensión, es un proceso gradual que requiere el contacto repetido con las mismas palabras en diferentes contextos y posiciones paradigmáticas. En otras palabras, para que una palabra se aprenda de manera incidental a partir de un contexto ésta debe encontrarse varias veces previamente (Jenkins et al. 1984; Webb 2007b) antes de que se pueda comprender y recordar el significado de un vocablo nuevo. En este sentido, parece que la metodología basada en contenidos permite recordar mejor el vocabulario al trabajar con un input oral y escrito que utiliza el mismo tema durante largos períodos de tiempo. Esta forma de trabajar 
los contenidos permite la repetición espaciada a lo largo de un tiempo prolongado, es decir, el encuentro y uso del mismo vocabulario en diferentes momentos y contextos.

Asimismo, las investigaciones de Wode (1999) sobre el aprendizaje incidental de vocabulario a través del estudio de disciplinas, como historia o geografía, demuestran que los alumnos sometidos al programa de inmersión lingüística adquieren más vocabulario, usan un vocabulario más rico y variado e incluyen más sinónimos que los demás grupos investigados. Estas conclusiones parecen corroborar los beneficios de la metodología basada en contenidos para el aprendizaje léxico ya que ofrece a los alumnos numerosas oportunidades de activar sus capacidades de aprendizaje. Los alumnos reciben gran cantidad de input oral y escrito, escuchan el input adicional procedente de las explicaciones del profesor y usan el vocabulario para interaccionar con los compañeros mientras resuelven problemas o realizan las actividades de clase.

Por otra parte, en su estudio sobre la adquisición incidental de vocabulario a partir del discurso oral, Brown, Sagers y LaPorte (1999) consideran que los alumnos conscientes de sus carencias léxicas aprenden el vocabulario con más facilidad, que las preguntas realizadas por el profesor facilitan la adquisición léxica y que el interés por un tema y la discusión continuada sobre el mismo favorecen la adquisición y el uso de gran cantidad de vocabulario. Todos estos aspectos están presentes en la metodología basada en el aprendizaje de contenidos ya que los alumnos trabajan sobre un tema que despierta progresivamente su interés, realizan tareas que ponen a prueba su competencia léxica y discuten de forma continuada sobre temas específicos, todo lo cual les obliga no sólo a adquirir, sino a usar de forma significativa grandes cantidades de vocabulario. El aprendizaje basado en contenidos permite aprender el léxico de forma incidental o fortuita ya que los discentes se centran principalmente en la comprensión del significado y no en aprender nuevos términos.

\section{INSTRUCCIÓN EXPLÍCITA DEL VOCABULARIO}

Neuber y Wilkins (2004) consideran que enseñar previamente el vocabulario desconocido, esencial para la comprensión del discurso, facilita la comprensión y proporciona una práctica inmediata que refuerza el aprendizaje ya que el alumno tendrá que usar ese vocabulario en sus respuestas o discusiones sobre los contenidos, todo lo cual contribuye a la retención del vocabulario a largo plazo. Otros investigadores (Coady y Huckin 1997; Schmitt y McCarthy 1997; Paribakht y Wesche 2000) afirman que el aprendizaje en contexto del vocabulario es más eficaz si éste se complementa con la instrucción léxica porque se agiliza el proceso de aprendizaje. Zimmerman (1997) propone, además, la instrucción léxica sistemática, mediante actividades interactivas y comunicativas. Por último, Thornbury (2002) recomienda la práctica espaciada y distribuida a lo largo de un período de tiempo para consolidar el vocabulario aprendido.

El método basado en contenidos permite enseñar el vocabulario específico asociado a los temas tratados debido a la prominencia que adquiere en el discurso, a los lazos semánticos que lo unen al tema o a otras palabras del texto y a que resulta necesario comprenderlo para entender el discurso. Este método concede, también, una enorme importancia al vocabulario académico, compartido por la mayoría de las disciplinas (Coxhead 2000), porque facilita la comprensión. Por este motivo, esta metodología puede ser de gran utilidad para trabajar 
este tipo de vocabulario que es más difícil de explicar, es polisémico y no es fácilmente deducible por el contexto. Debido a la gran cantidad de palabras específicas que se aprenden de forma incidental, el aprendizaje basado en contenidos permite dedicar más tiempo a la enseñanza explícita de vocabulario de tipo académico. De esta forma, será más fácil que los alumnos se fijen en ellas durante los repetidos encuentros y que sean capaces de aprender los diferentes significados de este tipo de léxico. Concretamente, algunos investigadores (Donley y Reppen 2001) resaltan la influencia de la metodología basada en contenidos en la adquisición de vocabulario académico con la ayuda de corpus lingüísticos. Estos autores recomiendan el uso de corpus lingüísticos para determinar la frecuencia del vocabulario académico y centrarse en la instrucción del léxico más frecuente.

Asimismo, el método basado en contenidos es muy adecuado para aprender familias léxicas puesto que al tratar un tema de forma continuada aumenta la oportunidad de encontrar palabras de la misma familia. El profesor puede explicar las diferentes palabras derivadas de la misma raíz, sus prefijos y sufijos de forma que el alumno pueda aprender a pensar en términos de familias léxicas y no en palabras aisladas. Por último, esta metodología permite enseñar los sinónimos de un mismo concepto de manera natural y estructurada. Al no disociar forma y contenido, induce al aprendizaje de diferentes formas de expresar un mismo concepto. Además, como este método obliga a parafrasear, reestructurar y resumir la información para hacerla más comprensible, el alumno entiende la importancia de conocer diferentes sinónimos que le permitan expresar los contenidos de forma diferente.

\section{LA ENSEÑANZA DE ESTRATEGIAS DE PROCESAMIENTO LÉXICO}

Otro aspecto a tener en cuenta es que para contribuir al aprendizaje autónomo se debe enseñar a los alumnos una serie de estrategias que les permitan deducir los significados ayudados por las pistas morfológicas o por el contexto, a utilizar técnicas de asociación de imágenes o de personalización que ayuden a recordar el léxico, a recordar expresiones que integren el vocabulario nuevo para aprender su uso, a relacionar palabras del mismo campo semántico, etc. Por este motivo, numerosos investigadores recomiendan la enseñanza de estrategias específicas que faciliten la adquisición del vocabulario a partir del contexto (Alyousef 2006; Oxford y Scarcella 1994; Fraser 1999; Coady 2000; Nation 2001). Schmitt (1997, 2000) considera, además, que la madurez cognitiva y la competencia lingüística influyen en el uso de estrategias de adquisición léxica y que éstas deberían introducirse progresivamente en las diferentes etapas del aprendizaje. En este sentido, la metodología basada en contenidos tiene en cuenta el proceso de maduración cognitiva y contribuye a que el alumno utilice una serie de estrategias de deducción y recuerdo del vocabulario que permiten un procesamiento mental más profundo y la integración del vocabulario aprendido en el sistema cognitivo.

Además, en línea con Nassaji (2003), que recomienda combinar el uso de determinadas estrategias con el uso de otras fuentes de información procedentes del texto o de fuera de él, la metodología basada en contenidos utiliza actividades que obligan al alumno a tener en cuenta la frase o el párrafo en el que se encuentra la palabra desconocida, a fijarse en la información extra-lingüística y a activar los conocimientos previos cuando intenta deducir el significado de una palabra. 
Por último, esta metodología suele utilizar textos con diagramas, gráficos, fotos, etc. que ayuden a la comprensión de las ideas y conceptos que se manejan. Estas imágenes visuales facilitan la comprensión y el recuerdo del léxico asociado ya que ayuda al cerebro a conectar lo abstracto con imágenes concretas. Son abundantes las investigaciones que demuestran que las técnicas de creación de imágenes visuales son enormemente eficaces para recordar el vocabulario a corto y largo plazo (Oxford y Crookall 1990).

\section{LA INTERACCIÓN ENTRE ALUMNOS MEDIANTE LAS ACTIVIDADES COMUNI- CATIVAS}

Algunos teóricos consideran que las actividades comunicativas pueden contribuir al aprendizaje de vocabulario debido a que favorecen la negociación sobre el léxico desconocido, proporcionan un contexto, ofrecen la oportunidad de que los alumnos presten atención a determinados términos al observar cómo sus compañeros los usan y, finalmente, permiten usar el léxico de manera productiva durante la actividad (Ellis y He 1999; Nation y Newton 2000; Webb 2005). Además, estos teóricos, partiendo de la hipótesis de la interacción de Long (1996), afirman que los intercambios verbales entre los alumnos no sólo contribuyen a modificar y hacer más comprensible el input, lo cual influye en el proceso de atención y retención del léxico desconocido, sino también contribuyen a modificar el output, facilitando la adquisición del vocabulario gracias al entrenamiento en el uso productivo del mismo. Como consecuencia, los alumnos se conciencian de sus carencias léxicas, reestructuran su repertorio léxico, utilizan los nuevos términos con mayor precisión y, además, desarrollan un conocimiento metalingüístico sobre el funcionamiento de la lengua meta. En este sentido, se podría afirmar que la metodología basada en el aprendizaje de contenidos es muy adecuada porque las actividades que se realizan suelen crear la necesidad de expresar determinadas ideas y conceptos que conciencian a los alumnos de sus lagunas léxicas, aumentando, así, la probabilidad de adquirir el léxico necesario para suplir esas carencias.

Como se viene diciendo, en la instrucción léxica parece que es mucho más útil utilizar técnicas inductivas que obliguen al alumno a elaborar y construir el significado de la palabra en lugar de ofrecérselo directamente sin que ellos realicen ningún esfuerzo cognitivo. Es evidente que involucrar a los alumnos de manera activa para descifrar los significados de los nuevos vocablos en un clima de colaboración con los compañeros puede ser enormemente provechoso (Nation y Newton 2000). El trabajo en grupo, propio de la metodología basada en el aprendizaje de contenidos, permite a los alumnos negociar el significado, solicitar las aclaraciones necesarias o comprobar su comprensión del léxico y, de esta forma, los alumnos pueden recibir un input modificado que facilita la comprensión de la información y que centra su atención en un vocabulario específico facilitando su aprendizaje. La colaboración entre los alumnos para determinar el significado del léxico nuevo es bastante eficaz ya que crea las condiciones ideales para la asimilación del mismo. Debido a que el aprendizaje se construye socialmente, este tipo de tareas interactivas proporcionan a los alumnos oportunidades de negociar y descubrir el significado del vocabulario con lo que se desarrolla su competencia léxica. En este sentido, cuando los alumnos sienten la necesidad de expresarse en inglés sobre los contenidos aprendidos de manera precisa y coherente se conciencian de la importancia de prestar atención a la forma y al contenido simultáneamente. Esto les obliga 
a reflexionar sobre sus carencias léxicas, a utilizar el nuevo léxico asociado a las ideas que quieren transmitir y a adquirir un conocimiento meta-lingüístico. El trabajo en grupo y el tipo de actividades de procesamiento integrado de forma y contenido instan al alumno a modificar su forma de expresarse para adquirir precisión y fluidez en el uso del vocabulario.

Por otra parte, hay lingüistas que consideran que es el tipo de actividades de procesamiento del input lo que más influye en la adquisición léxica. Según algunas investigaciones, la adquisición léxica está relacionada con el tipo de procesamiento cognitivo que se realiza durante el aprendizaje (Waring 2002). En opinión de Schmitt y Schmitt (1995), las actividades que exigen una mayor complejidad cognitiva durante la manipulación y procesamiento de una palabra contribuyen a su aprendizaje. La metodología basada en los contenidos permite a los alumnos realizar tareas desafiantes desde una perspectiva cognitiva y considerar forma y contenido simultáneamente, todo lo cual facilita la integración del vocabulario aprendido en el repertorio léxico del discente. Además, en consonancia con la teoría del grado de participación (involvement load theory) (Laufer y Hulstijn 2001), que afirman que la retención del vocabulario depende del grado de participación y del esfuerzo invertido, la metodología basada en contenidos consigue involucrar a los alumnos debido a que las actividades obligan a procesar previamente forma y contenido. Se podría afirmar, además, que esta metodología contribuye enormemente al aprendizaje de grandes cantidades de vocabulario debido a que las actividades de comprensión implican a los alumnos en el triple proceso de "necesidad", "búsqueda" y "evaluación" del vocabulario. De esta forma se consigue trabajar el léxico de forma dinámica y que el repertorio léxico de los alumnos vaya evolucionando y madurando durante la interacción verbal.

\section{LA ORGANIZACIÓN COGNITIVA}

Las teorías neurológicas sobre el funcionamiento del cerebro afirman que el cerebro necesita crear estructuras que hagan que la información resulte significativa. Los estudios sobre esquemas de conocimiento (Carrell 1987), también denominados mapas mentales de organización de la información, consideran que éstos permiten recordar mejor tanto los contenidos como el vocabulario utilizado para expresarlos. En cuanto a la adquisición léxica, la existencia de una estructura previa o nexo cognitivo al que asociar el vocabulario nuevo contribuye a una mejor retención y procesamiento. Se considera, por tanto, necesario que los alumnos relacionen el nuevo léxico con sus conocimientos previos porque crea anclajes cognitivos que permiten fijar mejor el vocabulario asociado a esos conceptos o conocimientos.

La metodología basada en los contenidos parte de la base de que para que se dé un verdadero aprendizaje es necesario ayudar a los alumnos a organizar la información y a crear estructuras que permitan entender, recordar y reproducir la información. En este sentido, la organización de la información en forma de diagramas o esquemas no sólo contribuye a comprender la información, sino que permite integrar mejor el vocabulario asociado a un tema dentro del repertorio léxico del alumno gracias tanto a la existencia de un contexto como a una estructura bien organizada. Este método utiliza mapas semánticos que integran el vocabulario dentro de las estructuras de significado ya existentes y conectan el vocabulario nuevo con las palabras ya conocidas. Parece aceptado que la creación de redes semánticas 
conectadas por el tema o el contexto favorece la adquisición del vocabulario. Este tipo de aprendizaje de una segunda lengua propone establecer conexiones entre significados mediante la solución de problemas o el desarrollo de razonamientos críticos y lógicos y, de esa forma, permite que el cerebro integre el uso del vocabulario con los contenidos aprendidos en determinados contextos.

\section{CONCLUSIONES}

En resumen, hay razones pedagógicas y lingüísticas que avalan el uso de una metodología basada en el aprendizaje de contenidos para reforzar y ampliar la competencia léxica. Esta metodología tiene en cuenta una serie de parámetros que favorecen el procesamiento de gran variedad de vocabulario dentro de diferentes contextos, proporciona información rica y variada sobre el vocabulario gracias a los repetidos encuentros, utiliza tareas comunicativas que contribuyen a adquirir cierta automaticidad y, por último, favorece la creación de asociaciones sintagmáticas y paradigmáticas que incrementan el repertorio léxico del alumno.

Por otra parte, como la finalidad que se persigue es adquirir contenidos a través de la lengua extranjera, se considera esencial la instrucción explícita del vocabulario con el fin de incrementar el repertorio léxico necesario para un mejor procesamiento del input. Debido a que para aprender los contenidos es necesario resumir, reestructurar y parafrasear la información procedente del input, esta metodología permite usar repetidamente el léxico de manera productiva y significativa. Se podría decir que el aprendizaje del léxico se da en espiral y no de forma lineal, ya que los alumnos elaboran, resumen y evalúan de forma crítica las ideas y los contenidos relacionados con un tema determinado utilizando repetidamente el mismo léxico. Como consecuencia, progresivamente profundizan en el conocimiento del vocabulario y aprenden a utilizarlo de forma más precisa. Además, esta metodología basada en el aprendizaje de contenidos suele utilizar textos con diagramas, gráficos, fotos, etc. que ayudan a procesar la información y a recordar el léxico.

El método basado en contenidos consigue cumplir los objetivos del verdadero aprendizaje léxico ya que se interesa por el proceso de aprendizaje que debe atravesar por las fases de atención, recuerdo y proceso generativo. Debido a que antes de leer o escuchar la información sobre el tema seleccionado se enseña previamente el vocabulario, los alumnos centran su atención en el léxico que deben aprender. Aprenden así estrategias de selección de la información y léxico necesario evitando desperdiciar su tiempo y esfuerzo en aprender léxico irrelevante para la comprensión. Posteriormente, durante el proceso de comprensión, tendrán repetidos contactos con el léxico seleccionado previamente en contextos significativos y variados. Durante este proceso receptivo, los alumnos se verán instados a recordar el significado de las palabras cada vez que ven o escuchan ese léxico desconocido. Consiguen así practicar estrategias de memorización y de deducción del significado. Finalmente, los alumnos tendrán que utilizar el léxico nuevo de manera productiva para responder a las preguntas planteadas, parafrasear o resumir la información, discutir sobre el tema o escribir las opiniones o reacciones sobre el tema tratado. Esta metodología supone una superación del concepto de competencia léxica como resultado de la mera repetición y memorización pasiva consiguiendo que el aprendizaje léxico se convierta en un proceso activo donde el alumno construya el significado y lo use en contextos significativos. De esta manera, en la 
que la forma y el contenido son indisociables, el aprendizaje léxico se hace de forma natural, lo que conlleva una integración más profunda y duradera en el sistema cognitivo del alumno.

\section{REFERENCIAS BIBLIOGRÁFICAS}

Adolphs, S. y N. Schmitt. 2003. "Lexical coverage of spoken discourse". Applied Linguistics 24, 4: 425-438.

Alyousef, H. S. 2006. “Teaching reading comprehension to ESL/EFL learners”. Journal of Language and Learning 5, 1: 63-73.

BARCROFT, J. 2004. "Effects of sentence writing in second language lexical acquisition". Second Language Research 20, 4: 303-334.

Brinton, D. M., M. A. Snow y M. B. Wesche. 2004. Content-Based Second Language Instruction. Ann Arbor: University of Michigan Press.

Bonk, W. J. 2000. "Second language lexical knowledge and listening comprehension". International Journal of Listening 14: 14-31.

Brown, C. 1995. "Factors affecting the acquisition of vocabulary: Frequency and saliency of words". Second Language Reading and Vocabulary Learning. Eds. T. Huckin, M. Haynes y J. Coady. Norwood, NJ: Ablex Publishing. 263-286.

Brown, C., S. L. Sagers y C. LaPorte. 1999. "Incidental vocabulary acquisition from oral and written dialogue journals". Studies in Second Language Acquisition 21: 259-283.

CARrell, P. 1987. "Content and formal schemata in ESL reading”. TESOL Quarterly 21, 3: 461-481.

COADY, J. 2000. "L2 vocabulary acquisition through extensive reading”. Second Language Vocabulary Acquisition. Eds. J. CoAdy y T. Huckin. Cambridge: Cambridge University Press. 225-237.

CoAdy, J. y T. Huckin, eds. 2000. Second Language Vocabulary Acquisition. Cambridge: Cambridge University Press.

Coxhead, A. 2000. “A New Academic Word List”. TESOL Quarterly 34, 2: 213-238.

DONLEY, K. M. y R. REPPEN. 2001. "Using corpus tools to highlight academic vocabulary in SCLT”. TESOL Journal 10, 2/3: 7-12.

Elley, W. y F. MangubHai. 1983. "The impact of reading on second language learning". Reading Research Quarterly 19: 53-67.

Ellis, R. y X. He. 1999. "The roles of modified input and output in the incidental acquisition of word meanings". Studies in Second Language Acquisition 21: 285-301.

FrASER, C.A. 1999. "Lexical processing strategy use and vocabulary learning through reading”. Studies in Second Language Acquisition 21: 225-241. 
Gass, S. 1999. "Discussion: Incidental vocabulary learning". Studies in Second Language Acquisition 21: 319-333.

Grabe, W. y F. Stoller. 2000. "Reading and vocabulary development in a second language”. Second Language Vocabulary Acquisition. Eds. J. COADy y T. Huckin. Cambridge: Cambridge University Press. 98-121.

Hu, M. y P. NAtion. 2000. "Vocabulary density and reading comprehension". Reading in a Foreign Language 8, 2: 689-96.

Huckin, T. y M. Haynes. 1995. "Summary and future directions". Second Language Reading and Vocabulary Learning. Eds. T. HuCKIN, M. HAYNES y J. COADY. Norwood, NJ: Ablex Publishing. 153-178.

Jenkins, J.R., M.L. Stein y K. Wysocky. 1984. "Learning vocabulary through reading". American Educational Research Journal 21: 767-787.

KRASHEN, S. 1989. "We acquire vocabulary and spelling by reading: Additional evidence for the Input Hypothesis". Modern Language Journal 73, 4: 440-463.

KruSE, A. F. 1979. "Vocabulary in context”. ELT Journal 33, 3: 207-213.

LAUfer, B. 2000. "The lexical plight in second language reading. Words you don't know, words you think you know, and words you can't guess". Second Language Vocabulary Acquisition. Eds. J. COADY y T. Huckin. Cambridge: Cambridge University Press. 20-34.

LAufer, B. y J. Hulstijn. 2001. "Incidental vocabulary acquisition in a second language: The construct of task-induced involvement". Applied Linguistics 21, 1: 1-26.

LoNG, M. 1996. "The role of linguistic environment in second language acquisition". Handbook of Language Acquisition. Eds. W. C. Ritchie y T. Bhatia. New York Academic Press. 413-668.

NASSAJ, H. 2003. 'L2 vocabulary learning from context: Strategies, knowledge sources, and their relationship with success in L2 lexical inferencing". TESOL Quarterly 37, 4: 645-670.

NATION, I. S. P. 1990. Teaching and Learning Vocabulary. Rowley, MA: Newbury House. 2001. Learning Vocabulary in Another Language. Cambridge: Cambridge University Press.

Nation, I. S. P. y J. Newton. 2000. "Teaching vocabulary". Second Language Vocabulary Acquisition. Eds. J. CoAdY y T. Huckin. Cambridge: Cambridge University Press: 238- 254.

Neuber, Gloria A. y E.A. Wilkins. 2004. Putting it All Together. The Directed Reading Lesson in the Secondary Content Classroom. Boston: Pearson Education.

Oxford, R. y D. Crookall. 1990. "Vocabulary learning: A critical analysis of techniques”. TESL Canada Journal 7, 2: 9-30. 
OXford, R. L. y R. C. SCARCELlA. 1994. "Second language vocabulary learning among adults: State of the art of vocabulary instruction". System 22, 2: 231-243.

ParibaKht, S. y M. Wesche. 2000. "Vocabulary enhancement activities and reading for meaning in second language vocabulary acquisition" Second Language Vocabulary Acquisition. Eds. J. CoAdy y T. Huckin. Cambridge: Cambridge University Press. 174-202.

Schmitт, N. 1997. "Vocabulary learning strategies". Vocabulary : Description, Acquisition, and Pedagogy. Eds. N. Schmitt y M. McCarthy. Cambridge: Cambridge University Press. 199-227. versity Press.

2000. Vocabulary in Language Teaching. Cambridge: Cambridge Uni-

Schmitt, N. y M. McCarthy, eds. 1997. Vocabulary: Description, Acquisition, and Pedagogy. Cambridge: Cambridge University Press

Schmitt, N. y P. CARTER. 2000. "The lexical advantages of narrow reading for second language readers". TESOL Journal 9, 1: 4-9.

Schmitт, N. y D. Sснмітт. 1995. "Vocabulary notebooks: Theoretical underpinnings and practical suggestions" ELT Journal 49, 133-143.

Stoller, F. y W. Grabe. 1995. "Implications for L2 vocabulary acquisition and instruction from L1 vocabulary research". Second Language Reading and Vocabulary Learning. Eds. T. Huckin, M. Haynes y J. Coady. Norwood, NJ: Ablex Publishing. 24-45.

Thornbury, S. 2002. How to Teach Vocabulary. Harlow: Longman.

WARING, R. 2002. "Basic principles and practice in vocabulary instruction". The Language Teacher. July. http://www.jalt-publications.org/tlt/articles/2002/07/waring (25 enero 2008).

WeBB, S. 2005. "Receptive and productive vocabulary learning: The effects of reading and writing on word knowledge". Studies in Second Language acquisition 27: 33-52.

. 2007a. "Learning word pairs and glossed sentences: The effects of a single context on vocabulary knowledge". Language Teaching Research 11, 1: 63-81.

.2007b. "The effects of repetition on vocabulary knowledge". Applied Linguistics 28, 1: 46-65.

Wode, H. 1999. "Incidental vocabulary acquisition in the foreign language classroom". Studies in Second Language Acquisition 21: 243-258.

ZimMERMAN, C.B. 1997. "Do reading and interactive vocabulary instruction make a difference? An empirical study". TESOL Quarterly 31, 1: 121-140. 